\title{
Number of Electrocardiogram Electrodes
}

National Cancer Institute

\section{Source}

National Cancer Institute. Number of Electrocardiogram Electrodes. NCI Thesaurus.

Code C154906.

The number of electrocardiogram electrodes used in the assessment. 